\title{
A Continuous Analysis Process Between Desktop and Collaborative Visual Analytics Environments
}

\author{
Dong Hyun Jeong* $\quad$ Evan Suma $^{\dagger} \quad$ Thomas Butkiewicz ${ }^{*} \quad$ William Ribarsky ${ }^{\S} \quad$ Remco Chang ${ }^{\text {Il }}$ \\ *Univ. of the District of Columbia \\ †niv. of Southern California \\ $\star+\$ \llbracket$ Univ. of North Carolina at Charlotte \\ IT Tufts University
}

\begin{abstract}
Since its inception, the field of visual analytics has undergone tremendous growth in understanding how to create interactive visual tools to solve analytical problems. However, with few exceptions, most of these tools have been designed for single users in desktop environments. While often effective on their own, most single-user systems do not reflect the collaborative nature of solving real-world analytical tasks. Many intelligence analysts, for example, have been observed to switch repeatedly between working alone and collaborating with members of a small team. In this paper, we propose that a complete visual analytical system designed for solving real-world tasks ought to have two integrated components: a single-user desktop system and a mirroring system suitable for a collaborative environment.
\end{abstract}

\section{INTRODUCTION}

In real-world analysis, domain experts often work together to solve analytical problems and have a beneficial to perform analysis together for complicated tasks. However, it has been observed that real-world analysts typically perform both individual and group tasks, and as a result must frequently transition between singleuser and multi-user collaborative workflows during the course of their analysis [1]. Despite this fact, most visual analytics solutions have been designed either as standalone single-user applications or as purely collaborative systems, and very few analytical tools have been developed to cohesively support both activities.

In this paper, we propose that a complete visual analytical system designed for solving real-world tasks ought to have two integrated components: a single-user desktop system and a mirroring system suitable for a collaborative environment. Our viewpoint begins with understanding limitations both in a single-user desktop environment and a collaborative environment. To demonstrate our viewpoint, we adapted an existing single-user desktop analytical tool for exploring data using principal component analysis (iPCA) into a collaborative touch-table system (iPCA-CE) using a user-centric approach. The system parameters and analytical findings for these tools are tightly integrated so that analysts may seamlessly transition back and forth between single-user and collaborative work environments without losing track of the analysis process.

Converting an existing single-user desktop application into a collaborative touch-table environment introduces unique technical challenges. The inherent differences between mouse and touchbased interaction require that the user interface must be redesigned, and limitations may also be imposed by factors such as system performance and screen resolution. However, despite the costs of

\footnotetext{
*e-mail:donghyunjeong@gmail.com

†e-mail:suma@ict.usc.edu

†e-mail:tjbutkie@uncc.edu

§e-mail:ribarsky@uncc.edu

IIe-mail:remcochang@gmail.com
}

converting an existing application into a new interaction modality, we believe that supporting both single-user and collaborative work in an integrated fashion provides important benefits for real-world analysis.

\section{System OVerview}

Although supporting collaboration when solving real-world analytical tasks is important, most visual analytics tools, with few exceptions, have been designed as single-user desktop systems. Multitouch surfaces support a rich set of interactions that allow multiple users to work together to solve complex analytical problems interactively. We selected the Interactive Principal Component Analysis (iPCA) application, which has been shown to be an effective and easy to use desktop visualization for analyzing data sets and interactively exploring the parameters of principal component analysis [2]. Figure 1(a) shows an example of a single user performing an analysis with iPCA and Figure 1(b) represents multiple users' collaborative analysis with the extended collaborative application (iPCA-CE).

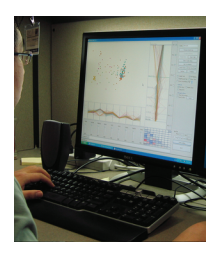

(a)

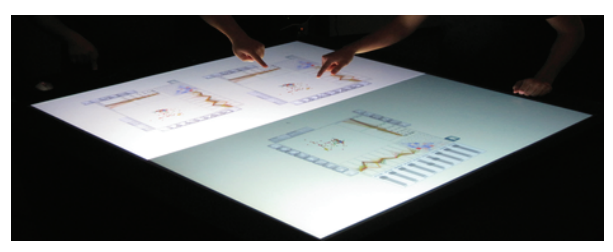

(b)
Figure 1: (a) A single user is performing an analysis of Glass dataset $(214 \times 9$ matrix) using iPCA. (b) Multiple users are interactively collaborating on a multi-touch table using iPCA-CE.

iPCA-CE is an extension of iPCA designed specifically for use in a collaborative touch-table environment. Each workspace in iPCA$\mathrm{CE}$ displays the same four views as iPCA. However, the system provides the capability to create multiple workspaces within the application, each of which can be used independently. A total of 18 touchable buttons were designed for interaction in iPCA-CE: nine buttons for interacting with represented data items, six buttons for controlling the application, one toggle button for expanding and collapsing the sliderbars panel, and two tab buttons for managing annotations and findings.

\subsection{Technical Challenges}

Creating a collaborative tabletop visual analytics system based on a single-user desktop counterpart has the following technical challenges: performance limitations, differences in rendering mechanisms, and differences in user input modalities. Together, iPCA and iPCA-CE form an integrated toolset which allows analysts to switch back and forth between the two visualizations on separate hardware without losing track of their current analysis tasks.

Performance In a collaborative environment, multiple processes and threads are necessary to manage the display and listen 
for incoming touch events. By utilizing a multi-process and multithreaded architecture, iPCA-CE becomes significantly more complex than iPCA. However, this architecture allows the application to take advantage of a multi-core CPU to support non-interrupted real-time interactivity for multiple users.

Rendering Mechanisms The rendering mechanism for iPCA-CE differs significantly from the desktop version since iPCA$\mathrm{CE}$ needs to support multiple interfaces for multiple users. While iPCA utilizes a single OpenGL context, iPCA-CE needs to create multiple "virtual" contexts, one for each of the interfaces. Since a multi-touch table is inherently without orientation, we wanted the iPCA-CE interface to be usable by all users standing around the table, regardless of their positions.

Input Modalities Another important interface difference between iPCA and iPCA-CE is that the "mouse-over" mechanism is no longer available on a touch-table. In traditional mouse-based interaction, the user can move a mouse over a visual element to highlight the element (such as to display its label) without the use of the mouse buttons. In a multi-touch environment that utilizes infrared refraction and reflection to detect a user's touch, there is no way to discern the difference between mouse-over and mouse-drag (holding down a mouse button and moving the mouse) because both operations require the user's finger to be touching the surface of the table.

\section{Understanding Users' Analytical Processes}

In a co-located collaborative environment (multi-touch table), numerous studies on collaborative visualization have been performed to overcome the limitations of sharing ideas, parallelizing efforts, and performing discussion and consensus building in single-user desktop environments. However, in a collaborative environment, such as our multi-touch table, a limited number of studies have been performed to find limitations, especially when solving analytical problems. We performed a user study of the collaborative iPCA-CE application with single, double, and multiple workspaces to understand how participants cooperate and share ideas in a collaborative environment.

Study Design A total of 12 people participated in the study (nine males, three females). The experiment required two participants to work together to solve a given task. The study used a within-subjects design with three conditions, corresponding to the number of available workspaces as Single (the two participants shared a single workspace), Double (each participant had their own personal workspace), and Multiple (participants were allowed to create as many workspaces as they desired). The order of the conditions was counterbalanced.

Study Results Each of the task performance measures was treated with a repeated measures ANOVA testing the withinsubjects effect of workspace condition. Although the completion time $(p=.28)$ and the verbal communication time $(p=.86)$ are not statistically significant across the condition, we find that the number of findings discovered across the condition was significant $(F(2$, $\left.10)=15.67, p<.01, \eta_{p}^{2}=.76\right)$. We conducted post-hoc analysis using paired-sample $t$-tests with a Bonferroni corrected significance value of $\alpha=.017$ to reduce error in multiple comparisons. Participants using a single workspace $(M=2.33, S D=1.03)$ discovered fewer findings than those using a double workspace $(M=5.83, S D$ $=2.32), p<.01$, or multiple workspaces $(M=5.17, S D=2.14)$, $p<.01$. The double workspace and multiple workspace conditions were not significantly different, $p=.42$. These results indicate that in a collaborative system, providing each user with their own personal workspace (or multiple workspaces) allows them to better perform an analysis task, although the task completion time and time spent communicating were not affected.
With regards to overall preferences for solving problems, most participants preferred the single workspace for communicating and sharing ideas. This indicates that a single shared workspace may be effective for communicating findings since users are looking at the same visual representation of the data. This is an interesting result, since creating a single, shared workspace was also possible in the multiple workspace condition, though participants may not have opted to do so.

Discussion From this study, we found that collaborative environments have advantages for solving analytical problems, especially when sharing ideas and findings. However, this approach has several limitations, such as:

- It is difficult to collaborate in a shared workspace when users' ideas conflict.

- Interference between collaborators may cause difficulty in forming new ideas or strategies.

- Users become fatigued when standing at a multi-touch table for long periods of time.

In light of both the advantages and limitations of collaborative environments, we suggest that users' analyses should not be isolated in one environment (see Figure 2). Instead, the general analytical process that should be supported and maintained to allow analysts to switch back and forth between single-user and collaborative workflows.

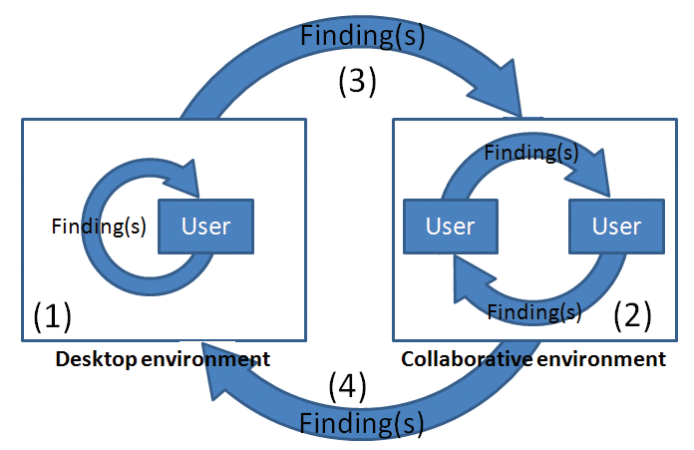

Figure 2: An illustration of users' processes of solving complex analytical problems and sharing analysis results (findings), including (1) asynchronous self-sharing in a desktop environment, (2) synchronous sharing in a collaborative environment, and ( 3 and 4 ) asynchronous transitional sharing processes between environments.

\section{Conclusion}

In this paper, we presented an integrated visual analytics toolset composed of a single-user desktop application and a collaborative touch-table system. Based on a study of users' analytical processes, we presented an informal model for the general analytical process that occurs as analysts switch back and forth between single-user and collaborative environments. Finally, putting this model into practice, we presented our improvements to both the desktop and collaborative applications for supporting this continuous analysis process.

\section{References}

[1] G. Chin, Jr., O. A. Kuchar, and K. E. Wolf. Exploring the analytical processes of intelligence analysts. In $\mathrm{CHI}$ '09: Proceedings of the 27th international conference on Human factors in computing systems, pages 11-20, New York, NY, USA, 2009. ACM.

[2] D. H. Jeong, C. Ziemkiewicz, B. Fisher, W. Ribarsky, and R. Chang. ipca: An interactive system for pca-based visual analytics. Computer Graphics Forum (EuroVis 2009), 28(3):767-774, 2009. 